\title{
Focalização da Atenção Visual
}

\author{
Visual Attention Focusing
}

\author{
Joaquim Carlos Rossini ${ }^{*}, a$ \& Cesar Alexis Galera ${ }^{b}$ \\ ${ }^{a}$ Universidade Federal de Uberlândia \& ${ }^{b}$ Universidade de São Paulo
}

\begin{abstract}
Resumo
Neste estudo investigamos o ajuste dinâmico do foco atentivo em função de algumas características físicas de uma dica visual exógena. O primeiro experimento investigou o efeito da área delimitada por uma dica visual apresentada por diferentes intervalos entre estímulos (IEE). O segundo experimento investigou a disponibilidade dos recursos atentivos no meridiano horizontal e vertical do campo visual e o efeito de letras flanqueadoras apresentadas dentro e fora do foco atentivo. Os resultados do primeiro experimento sugerem que o foco da atenção pode ser mantido estável por um período superior a $500 \mathrm{~ms}$, e que o tempo pelo qual os recursos atentivos podem permanecer estáveis é inversamente proporcional a sua área. Estes resultados também confirmam uma relação inversa entre o tamanho da área a ser atendida e a eficiência no processamento da informação. Os resultados do segundo experimento corroboram o efeito causado por letras flanqueadoras observado por outros autores (B. Eriksen \& Eriksen, 1974; Miller, 1991), com um aumento do tempo de reação na presença da letra flanqueadora incompatível com o alvo. As letras flanqueadoras têm um efeito significativamente menor quando apresentadas fora da área delimitada pela dica visual. Este resultado indica uma ação atentiva inibitória fora da área de focalização. Além disso, observou-se uma possível assimetria na distribuição dos recursos inibitórios entre o meridiano horizontal e vertical do campo visual.

Palavras-chave: Atenção visual; Foco atentivo; Tempo de reação.
\end{abstract}

\begin{abstract}
This study investigated the dynamic adjustment of the attention focusing in function of some physical characteristics of an exogenous visual cue. The first experiment investigated the effect of the area delimitated by a visual cue presented by different intervals between stimuli. The second experiment investigated the availability of attentional resources in the horizontal and vertical meridian of the visual field and the effect of flanking letters presented in and out of the attention focusing. The results of the first experiment suggest that the attention focusing may be kept stable for a period longer than 500 milliseconds and that the time through which the attention resources can remain stable is inversely proportional to their area. These results also corroborated an inverse relation between the size of the area to be fulfilled and the information processing efficiency. The results of the second experiment corroborated the effect caused by flanking letters observed by other authors (B. Ericksen \& Ericksen, 1974; Miller, 1991) with an increase on the reaction time in the presence of the flanking letter incompatible with the target. Flanking letters have a significant smaller effect when presented out of the area delimitated by the visual cue. This result indicates an inhibitory attentional action out of the focusing area. Moreover, a possible asymmetry in the distribution of these inhibitory resources between the horizontal and vertical meridian of the visual field was also observed.

Keywords: Visual attention; Attention focusing; Reaction time.
\end{abstract}

Sempre que procuramos um objeto em uma prateleira, lemos um texto, ou andamos despreocupadamente por uma rua movimentada, nosso sistema cognitivo seleciona e processa estímulos visuais de maneira atentiva. Neste sentido, o termo "atenção" é amplamente utilizado pela psicologia experimental humana para definir uma grande variedade de processos mentais relacionados à manutenção do estado geral de alerta e vigília, seleção da in-

${ }^{*}$ Endereço para correspondência: Universidade Federal de Uberlândia, Rua Pará, Umuarama, Uberlândia, MG, Brasil, CEP 14040-901. E-mail: jrossini@fapsi.ufu.br formação relevante, manutenção ativa da informação na memória de trabalho, bem como relacionados aos processos de ajuste dinâmico dos recursos atentivos no campo visual.

Particularmente, acerca dos processos que envolvem a focalização, ou o ajuste dinâmico de recursos atentivos no campo visual, C. Eriksen e St. James (1986) e Posner, Snyder e Davidson (1980) apresentaram dois modelos, bastante parecidos, para explicar o processamento dos objetos apresentados em uma cena visual. O modelo proposto por Posner et al. (1980) preconiza que os recursos atentivos são focalizados em uma dada localização espacial como um "holofote" que atende a uma área pouco 
flexível, ao passo que C. Eriksen e St. James (1986) preconizam que a focalização dos recursos atentivos pode ser ajustada conforme a área dos estímulos a serem processados, atuando desta forma como o "zoom" de uma lente de aumento. De acordo com este último modelo (modelo da lente zoom), há uma relação inversa entre a eficiência e a eficácia do processamento da informação em relação ao tamanho da área a ser focalizada. Tal afirmação vem sendo sistematicamente corroborada em várias investigações que demonstram mais claramente a relação entre o tamanho da área a ser atendida e a eficiência no processamento da informação nela contida, em um efeito conhecido como efeito do tamanho da dica (Benso, Turatto, Mascetti, \& Umiltà, 1998; Castiello \& Umiltà, 1990; Galera \& von Grünau, 2003; Maringelli \& Umiltà, 1998; Turatto et al., 2000).

Benso et al. (1998) investigaram o tempo necessário para a efetivação do processo de focalização em uma área específica do campo visual, bem como o tempo que esta focalização pode ser mantida pelo sistema. Os autores manipularam não apenas o tamanho da área a ser atendida (dica visual da área a ser focalizada), mas também o intervalo entre a apresentação da dica e do estímulo alvo (intervalo entre estímulos - IEE). De modo geral, o resultado destes autores confirma o efeito do tamanho da dica e sugere que o processo de focalização pode ocorrer em intervalos muito breves (entre 33 e $66 \mathrm{~ms}$ ) e permanecer estável por um período próximo a $500 \mathrm{~ms}$. Além deste intervalo de exposição, a focalização da atenção na área delimitada pela dica não pode ser mais mantida e os recursos voltam a um estado difuso.

Outro aspecto interessante acerca do processo de focalização dos recursos atentivos foi investigado por Facoetti e Molteni (2000) e aponta para um possível mecanismo atentivo inibitório fora da área focalizada. Estes autores manipularam a extensão do foco atentivo utilizando uma dica espacial circular pequena $\left(3^{\circ} \mathrm{de}\right.$ ângulo visual) ou grande ( $9^{\circ}$ de ângulo visual), apresentada por um IEE de $0 \mathrm{~ms}$ ou $500 \mathrm{~ms}$. Logo após este período de apresentação, um ponto vermelho ou verde, definido como alvo era apresentado no centro da dica. $\mathrm{O}$ alvo era sempre flanqueado horizontalmente por dois pontos, apresentados a uma distância constante do alvo central. Estes estímulos flanqueadores apresentavam uma cor compatível ao alvo (alvo verde - estímulos flanqueadores verdes ou alvo vermelho - estímulos flanqueadores vermelhos) ou incompatível (alvo verde estímulos flanqueadores vermelhos ou alvo vermelho estímulos flanqueadores verde). A tarefa dos participantes era identificar, o mais rápido possível, a cor do alvo, pressionando uma tecla do computador. Como os estímulos flanqueadores eram apresentados sempre na mesma posição, nas provas com a dica pequena os flanqueadores eram apresentados fora da área da dica, enquanto que nas provas com a dica grande, estes estímulos eram apresentados dentro da área da dica. De acor- do com B. Eriksen e Eriksen (1974), o tempo de reação em tarefas com estímulos flanqueadores incompatíveis é sempre maior do que o tempo de reação observado em provas com os estímulos flanqueadores compatíveis ou neutros, o que sugere um efeito deletério na análise do estímulo alvo causado pela presença dos estímulos incompatíveis. Os resultados observados por Facoetti e Molteni (2000) mostraram que o efeito dos estímulos flanqueadores apresentados dentro da área delimitada pela dica era maior do que quando estes estímulos eram apresentados fora da dica visual. Quando os estímulos e a dica eram apresentados simultaneamente (IEE $=0 \mathrm{~ms}$ ), o efeito negativo do estímulo flanqueador incompatível foi significativamente maior. Quando a dica permanecia exposta por um período de $500 \mathrm{~ms}$ antes da apresentação dos estímulos (IEE $=500 \mathrm{~ms}$ ) o efeito negativo dos estímulos flanqueadores incompatíveis apresentados fora da área delimitada pela dica diminuiu significativamente. Os autores interpretaram esse resultado como uma possível evidencia da ação inibitória da atenção fora da área delimitada pela dica no meridiano horizontal do campo visual. Todavia, um aspecto não explorado pelos autores foi uma possível assimetria destes processos em relação ao meridiano vertical no qual a informação irrelevante pode ser apresentada.

O modelo proposto por C. Eriksen e colaboradores (C. Eriksen \& St. James, 1986; C. Eriksen \& Yeh 1985) sugere que o foco da atenção apresenta um formato circular, ou oval, muito flexível quanto ao tamanho, mas pouco flexível quanto à sua forma de distribuição pelo campo visual. Usai, Umiltà, e Nicoletti (1995), por exemplo, mostraram que os participantes de um experimento de focalização atentiva são capazes de mobilizar a atenção sobre uma área demarcada por uma dica visual exógena, mas são incapazes de ignorar voluntariamente a informação apresentada dentro de uma área específica dentro da dica. De acordo com esses autores, este tipo de resultado apóia a idéia de um foco atentivo com um formato circular unitário, muito pouco flexível quanto ao seu formato. Por outro lado, alguns estudos apontam a possibilidade de uma maior flexibilidade na distribuição do foco atentivo quanto a este aspecto. Por exemplo, estudos conduzidos por Egly e Homa (1984) e Juola, Bouwhuis, Cooper e Warner (1991) sugerem que o foco da atenção pode ser mobilizado sobre regiões concêntricas do campo visual, ajustando-se à áreas anelares do campo visual, o que sugere a possibilidade de uma capacidade de ajuste muito ampla e que pode abranger vários aspectos do processo dinâmico de ajuste, que incluem o tempo necessário para o ajuste do foco atentivo por processos excitatórios e inibitórios, respectivamente, dentro e fora do foco, e a possibilidade de uma distribuição assimétrica de recursos ao longo dos meridianos do campo visual.

No entanto, vários estudos têm sugerido que, em certas circunstâncias, a área focalizada pelos recursos atentivos pode assumir uma forma mais flexível, defini- 
da tanto de maneira endógena quanto exógena (Galera, von Grünau, \& Panagopoulos, 2005; Panagopoulos, von Grünau, \& Galera, 2004). E, de maneira mais geral, alguns estudos têm sugerido uma assimetria na distribuição dos recursos atentivos em relação aos quatro quadrantes do campo visual. Sanders e Brück (1991), por exemplo, apontam que os recursos atentivos podem estar mais concentrados ao longo do meridiano horizontal, no quadrante leste, e ao longo do meridiano vertical, no quadrante norte, do campo visual.

No presente estudo investigamos alguns aspectos do processo dinâmico de focalização atentiva em regiões delimitadas do campo visual. O primeiro experimento procurou determinar o tempo necessário para o ajuste do foco da atenção em uma tarefa de discriminação simples. O segundo experimento investigou a distribuição dos recursos atentivos dentro e fora do foco da área atendida, levando em conta o meridiano vertical e horizontal do campo visual. Neste segundo experimento, a distribuição dos recursos atentivos foi investigada através do efeito de letras flanqueadoras (flankers) compatíveis e incompatíveis no tempo de reação para a discriminação do alvo (B. Eriksen \& Eriksen, 1974). Todos os procedimentos experimentais realizados neste trabalho foram aprovados pelo Comitê de Ética em Pesquisa - CEP.

\section{Experimento 1}

\section{Método}

\section{Participantes}

Este experimento contou com a participação voluntária de treze sujeitos, quatro do sexo masculino e nove do sexo feminino, com idades entre 21 e 30 anos (idade média de 25 anos e desvio-padrão $=2,5$ ). Dois dos participantes eram canhotos e todos os participantes apresentavam visão normal ou corrigida.

\section{Material e Estímulos}

Os estímulos foram apresentados em um monitor LG Flatron 795 FT plus de 19 polegadas, com varredura da tela ajustada para $60 \mathrm{~Hz}$, acoplado a um microcomputador com processador Pentium e placa de vídeo Trident 9440 PCI. O controle da apresentação dos estímulos e o registro do tempo de resposta foram realizados pelo utilitário E-Prime 1.0 (Schneider, Eschman, \& Zuccoloto, 2002). Os participantes mantiveram os olhos a uma distância aproximada de $57 \mathrm{~cm}$ da tela de apresentação sem restrições aos movimentos da cabeça. Os estímulos foram apresentados em branco com uma luminosidade de $56 \mathrm{~cd} / \mathrm{m}^{2}$ sobre um fundo preto com uma luminosidade de $0,05 \mathrm{~cd} /$ $\mathrm{m}^{2}$. O alvo era uma letra $\mathrm{O}$ ou W com $0,6 \times 0,4$ graus de ângulo visual, sempre apresentada no centro da tela. A dica era um quadrado cujos lados subentendiam dois graus de ângulo visual (dica pequena) ou oito graus de ângulo visual (dica grande), apresentados sempre no cen- tro da tela. As margens da dica visual apresentavam a mesma luminância das letras alvos. Todas as provas eram iniciadas com a apresentação de um pequeno sinal de soma (+) com 0,5 x 0,5 graus de ângulo visual (fixação). A seqüência de eventos e os estímulos utilizados estão representados esquematicamente na Figura 1.

A

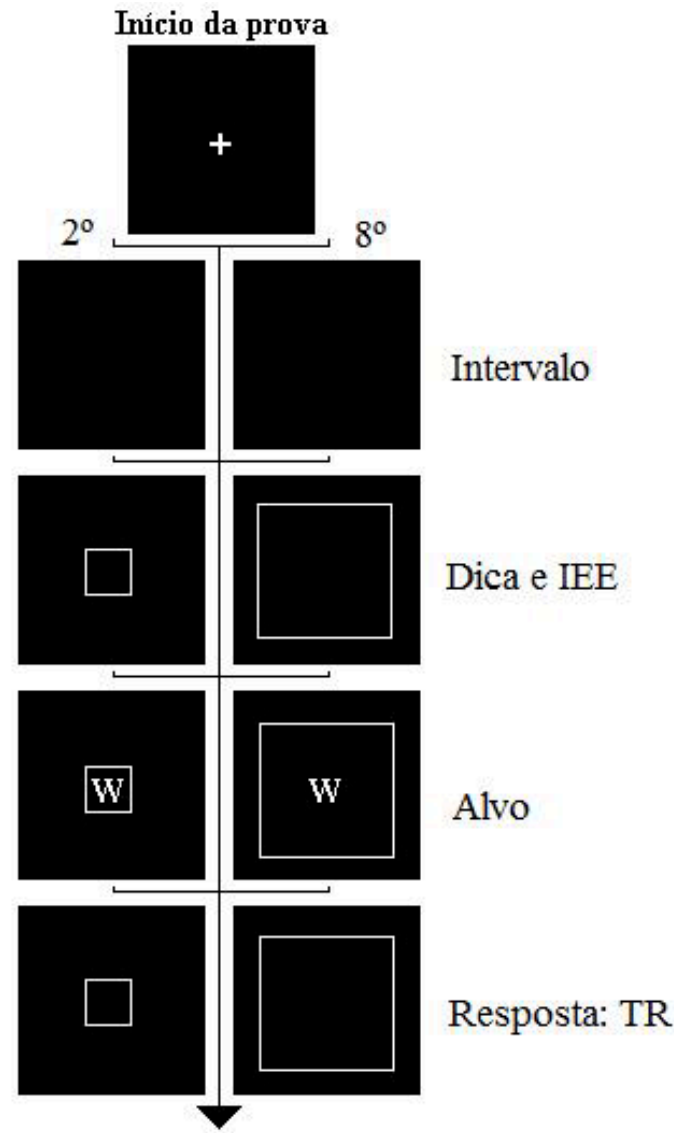

Fim da prova

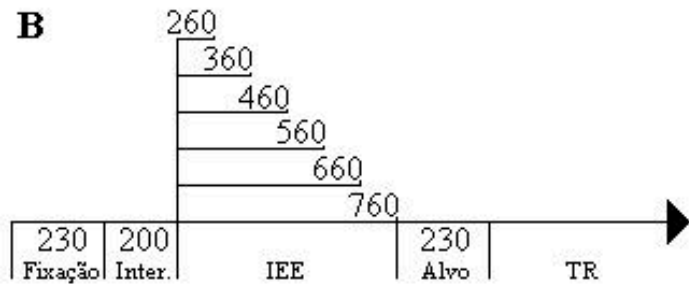

Figura 1. A- Representação esquemática dos estímulos apresentados no Experimento 1. A representação mostra a seqüência de eventos em cada prova (dicas: 2 ou 8 graus de ângulo visual). B- Sequiência temporal (ms) de eventos em cada prova do experimento

\section{Procedimento}

Cada prova tinha início com a apresentação do sinal de fixação por $230 \mathrm{~ms}$, seguido por um intervalo de 200 ms, no qual nenhum estímulo era apresentado. Após este intervalo, a dica era apresentada no centro da tela permanecendo até o fim da prova. A letra alvo era apresentada sempre na mesma posição no centro da dica e da tela do monitor. A tarefa do participante era discri- 
minar a letra $\mathrm{O}$ ou $\mathrm{W}$ apresentada no centro da dica visual. A discriminação da letra alvo era registrada quando o participante pressionava a tecla respectiva $(\mathrm{O}$ ou W) no teclado do computador. Estas respostas eram dadas com os dedos indicadores direito (letra $\mathrm{O}$ ) e esquerdo (letra W).

A dica era apresentada por um intervalo entre estímulos (IEE) que podia variar em 260, 360, 460, 560, 660 ou $760 \mathrm{~ms}$. Após este intervalo, o alvo era apresentado por $230 \mathrm{~ms}$. A dica permanecia até a conclusão da prova. Imediatamente após a resposta, o participante era informado sobre a sua precisão através de um sinal azul (resposta correta) ou vermelho (resposta incorreta), semelhante ao sinal de fixação apresentado no início da prova. Nas provas não respondidas o sinal era apresentado em branco. Os participantes eram instruídos a responder o mais rápido possível sem cometer erros. Cada sujeito participou de uma sessão experimental com 480 provas, 40 provas por tratamento (6 IDEs x 2 tamanhos da dica), apresentadas de maneira aleatória.

\section{Resultados}

A análise dos dados levou em consideração apenas os tempos de reação (TR) obtidos em provas com resposta correta, o que ocorreu em $96,4 \%$ das vezes. A porcentagem de erros cometidos não apresentou diferença significativa em nenhuma das condições experimentais. Os TRs que desviaram da média, mais do que dois desvios padrão, em cada condição experimental, foram eliminados da analise. Foi realizada uma ANOVA para medidas repetidas com os TRs médios de cada participante em função do tamanho da dica ( 2 ou 8 graus), e do intervalo entre estímulos IEE (260, 360, 460, 560, 660, $760 \mathrm{~ms})$. Esta análise mostrou um efeito significativo para o tamanho da dica $\left(F(1,12)=30,57, p<0,01 ; \eta^{2}=0,72\right)$, com TRs mais rápidos com a apresentação da dica

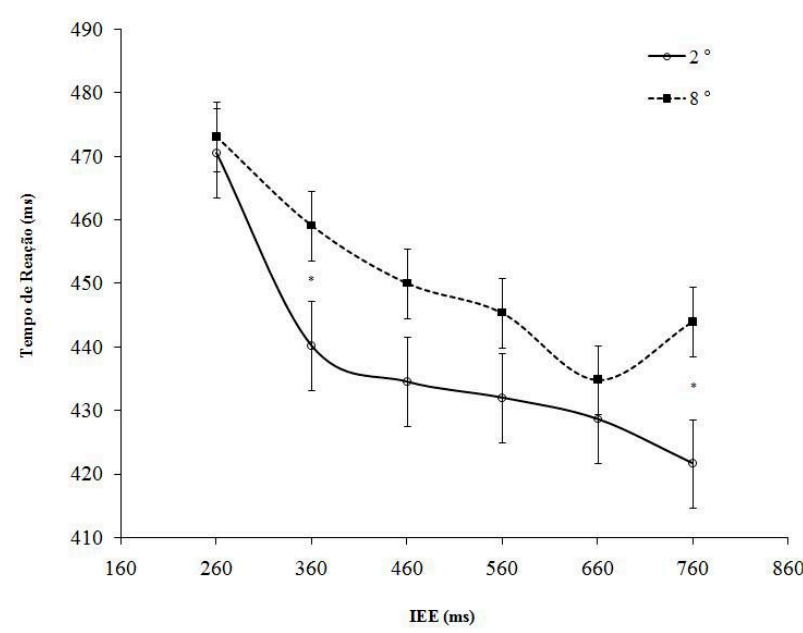

Figura 2. Tempo de reação médio (TR) em função do tamanho da dica visual ( 2 ou 8 graus de ângulo visual) e do intervalo entre estímulos (IEE). $\mathrm{O}$ asterisco indica as diferenças significativas $(p=0,05)$ pequena $\left(2^{\circ}\right)$ do que os apresentados com a apresentação da dica grande $\left(8^{\circ}\right)$. O fator IEE apresentou um efeito significativo no TR $\left(F(1,12)=32,70, p<0,01 ; \eta^{2}=0,73\right)$, com uma relação inversa entre o tempo de IEE e o tempo de resposta dos participantes. A Figura 2 mostra a média do tempo de reação dos participantes em função do tamanho da dica visual e do intervalo entre estímulos (IEE). Um teste post hoc (Newman-Keuls) confirmou que as diferenças entre o tamanho da dica ocorrem significativamente no IEE $360 \mathrm{~ms}$ (diferença na média dos TRs= $19 \mathrm{~ms}, p=0,01(d=0,46)$ e no IEE $760 \mathrm{~ms}$ (diferença na média dos TRs $=21 \mathrm{~ms}, p=0,01(d=0,51)$.

\section{Discussão}

Os resultados observados sugerem que os recursos atentivos foram ajustados à área delimitada pela dica visual e que esse processo foi compatível ao modelo proposto por C. Eriksen e colaboradores (C. Eriksen \& St. James, 1986; C. Eriksen \& Yeh 1985). De acordo com este modelo o efeito do tamanho da dica pode ser atribuído à distribuição dos recursos atentivos dentro da área delimitada pelas bordas da dica.

Neste processo, quanto menor a área a ser atendida, maior a eficiência do processamento da informação. Os resultados mostram um ajuste rápido dos recursos à área delimitada pela dica visual. Nesta condição de discriminação simples, o efeito do tamanho da dica foi observado após um IEE de $360 \mathrm{~ms}$. Uma possível interpretação para este ajuste, relativamente tardio dos recursos atentivos à dica visual, pode ser sugerida levando-se em consideração a característica da tarefa de discriminação que talvez exija recursos atentivos distintos dos exigidos em uma tarefa de detecção simples, como a utilizada por Benso et al. (1998) ou em uma tarefa de busca visual (Galera et al., 2005). Neste sentido, a tarefa de discriminação simples pode exigir um ajuste atentivo mais elaborado e, portanto, mais tardio no processamento da informação, o que poderia contribuir para um intervalo extenso para o ajuste dos recursos a uma área específica. Assim, o uso de letras como estímulo alvo pode ter contribuído para o ajuste relativamente lento do foco atentivo. Esta possível característica será investigada em trabalhos futuros.

Outro aspecto interessante observado neste experimento foi que o recurso atentivo tende a permanecer estável por mais tempo em áreas menores em relação a áreas maiores, como mostra a inversão da tendência no tempo de reação após o IEE $660 \mathrm{~ms}$. Isto pode sugerir um efeito do tamanho da dica, não apenas na eficiência no processamento da informação, mas também no tempo que estes recursos podem permanecer estáveis em uma determinada área. Assim, uma possibilidade é que o tamanho da área focalizada apresente uma relação inversa ao tempo que este foco pode ser mantido estável no campo visual, uma suposição que também será objeto de investigação. 


\section{Experimento 2}

O Experimento 2 foi delineado para investigar a distribuição dos recursos atentivos dentro e fora da área de focalização. Para tanto, foi adotado um paradigma de letras flanqueadoras, compatíveis e incompatíveis com a letra alvo (B. Eriksen \& Eriksen, 1974). Esta tarefa exige que o participante discrimine uma letra alvo flanqueada por letras compatíveis ou incompatíveis. O participante é solicitado a identificar a letra central de um arranjo de três letras. Por exemplo, a letra alvo pode ser a letra "O" ou "W" apresentada em um arranjo compatível (OOO) ou incompatível (WOW). Invariavelmente, o tempo de reação é maior quando as letras flanqueadoras são incompatíveis com o alvo. Este efeito é interpretado como o resultado de um processo de competição pela resposta baseada no espaço. Esta interpretação é bastante adequada uma vez que o efeito deletério no tempo de reação diminui com o aumento da distância entre a letra alvo e as letras flanqueadoras incompatíveis (Miller, 1991).

No presente estudo as letras flanqueadoras foram apresentadas dentro ou fora da área delimitada pela dica, com uma orientação horizontal ou vertical no campo visual. Esta dica visual podia apresentar uma área pequena que circundava apenas a letra alvo (condição controle), ou uma área maior vertical ou horizontalmente orientada. Em aproximadamente $67 \%$ das provas as letras flanqueadoras eram apresentadas fora da dica visual. Assim, caso a dica exerça efetivamente um papel na delimitação e na orientação dos recursos atentivos no campo visual, o efeito deletério provocado pelas letras flanqueadas incompatíveis dentro e fora da área delimitada será distinto, apesar da distância entre as letras flanqueadas e a letra alvo permanecer constante.

\section{Método}

\section{Participantes}

Este experimento foi realizado pelos mesmos treze participantes que realizaram o Experimento 1 .

\section{Material e Estímulos}

O equipamento utilizado neste experimento, os estímulos alvo, o sinal de fixação, os sinais de precisão da prova e as condições de execução foram iguais as do Experimento 1. A dica visual apresentava as seguintes dimensões: dica controle (1 x 1 grau de ângulo visual); dica orientada ( $1 \times 16$ graus de ângulo visual). $\mathrm{O}$ alvo era uma letra $\mathrm{O}$ ou $\mathrm{W}$ apresentada sempre no centro da tela e da dica visual. A letra alvo era ladeada por duas letras flanqueadoras apresentadas a uma distância constante de 1,2 graus de ângulo visual. Estas três letras formavam um arranjo vertical ou horizontal. As letras flanqueadoras pertenciam ao mesmo universo das letras alvo, $\mathrm{O}$ ou W, e eram apresentadas de maneira compa- tível ou incompatível com a mesma, como exemplificado na Figura 3A.
A

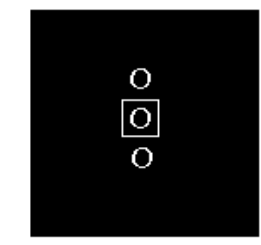

2

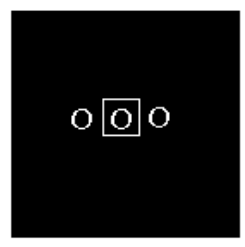

3

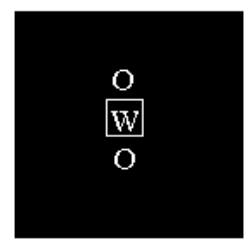

4

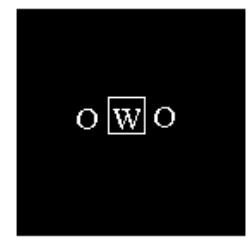

(B)
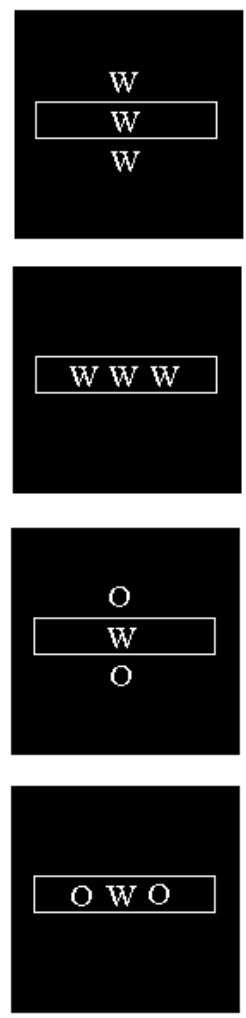

(C)
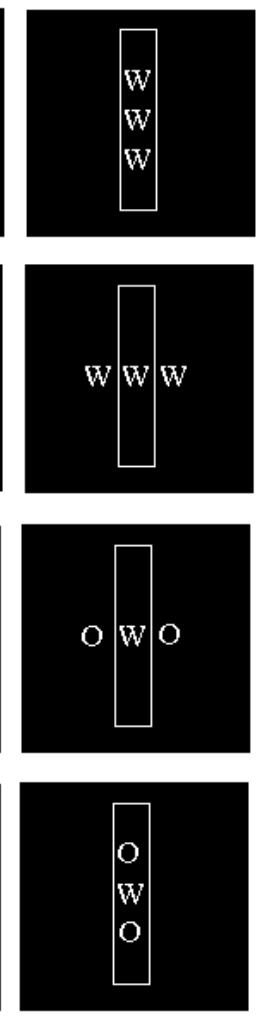

B

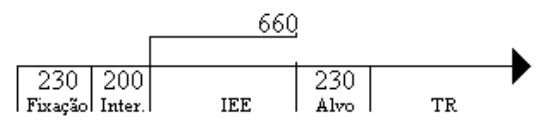

Figura 3. A- Representação esquemática dos estímulos apresentados no Experimento 2. B- Seqüência de eventos apresentados em cada prova. As colunas A, B e C, mostram exemplos das dicas visuais, respectivamente, dica controle, dica horizontal e dica vertical. As linhas 1, 2, 3 e 4, mostram os possíveis arranjos apresentados, respectivamente, letras compatíveis na vertical, letras compatíveis na horizontal, letras incompatíveis na vertical e letras incompatíveis na horizontal

\section{Procedimento}

A tarefa do participante era discriminar a letra central do arranjo pressionando a tecla correspondente no teclado do computador, tecla "W" ou "O". Cada prova tinha início com a apresentação do sinal de fixação por $230 \mathrm{~ms}$, seguido por um intervalo de $200 \mathrm{~ms}$, no qual nenhum estímulo era apresentado. Após este intervalo, um quadrado ou um retângulo era apresentado no centro da tela até o final da prova. Após um intervalo de $660 \mathrm{~ms}$, a letra alvo (O ou W) e as letras flanqueadoras eram apresentadas simultaneamente sempre no centro da tela de apresentação por $230 \mathrm{~ms}$. O sujeito dispunha de $1020 \mathrm{~ms}$ para efetuar a resposta. Os participantes 
foram informados sobre a precisão de cada resposta como nos experimentos anteriores. A Figura 3B mostra a seqüência temporal de eventos durante uma prova. Foram manipulados três fatores experimentais: tipo de dica (controle, horizontal, vertical), orientação dos estímulos (horizontal ou vertical) e compatibilidade entre as letras flanqueadoras e a letra alvo (compatível ou incompatível). Cada condição foi apresentada 20 vezes por bloco, de maneira aleatória, totalizando 240 provas por bloco. Cada participante foi submetido a quatro blocos totalizando 960 provas por participante. Em $66,6 \%$ das provas a letra franqueadora compatível ou incompatível era apresentada fora da área delimitada pela dica visual.

\section{Resultados}

A análise dos resultados levou em consideração apenas os tempos de reação (TR) obtidos nas provas com resposta correta $(95,7 \%)$ e que não excederam dois desvios padrão além da média do TR específico em cada condição experimental. A porcentagem de erros cometidos não apresentou diferença significativa em nenhuma das condições experimentais. A análise (ANOVA para medidas repetidas) mostrou um efeito significativo no tempo de reação associado ao tipo de dica (controle, horizontal, vertical $)\left(F(1,12)=29,58 ; p<0,05 ; \eta^{2}=0,71\right)$, à orientação dos estímulos (horizontal, vertical) $(F(1$, $\left.12)=6,37 ; p<0,05 ; \eta^{2}=0,34\right)$ e à compatibilidade entre alvo e letras flanqueadoras (compatível, incompatível) $\left(F(1,12)=47,65 ; p<0,05 ; \eta^{2}=0,80\right)$. Houve uma interação significativa entre os fatores tipo de dica e orientação dos estímulos $\left(F(2,24)=5,60 ; p<0,05 ; \eta^{2}=\right.$ 0,32 ) e uma tripla interação entre os fatores $F(2,24)=$ 6,$84 ; p<0,05 ; \eta^{2}=0,36$. As demais interações entre os fatores não foram significativas.

Uma análise realizada apenas com os flanqueadores compatíveis apresentados fora da dica controle e das dicas orientadas vertical e horizontalmente confirmou um efeito no TR atribuído ao tamanho da dica $F(1,12)=$ 27,79; $p<0,01 ; \eta^{2}=0,70$. Esta análise, que permitiu comparar uma dica menor (controle) com dicas maiores (orientadas vertical e horizontalmente), mostrou que a dica controle é acompanhada de um TR menor (TR médio $=459 \mathrm{~ms}$ ) do que a dica orientada (TR médio= $475 \mathrm{~ms}$ ).

Uma análise realizada apenas com as respostas obtidas na condição controle mostrou que o tempo de reação foi afetado pela compatibilidade entre alvo e letras flanqueadoras, com tempos menores para letras flanqueadoras compatíveis (TR médio $=459 \mathrm{~ms}$ ) do que para letras flanqueadoras incompatíveis, $($ TR médio $=476 \mathrm{~ms})$ $\left(F(1,12)=17,58 ; p<0,01 ; \eta^{2}=0,59\right)$. Ainda nesta condição, os participantes foram mais eficientes na discriminação da letra alvo apresentada em meio a um arranjo vertical (TR médio $=464 \mathrm{~ms}$ ), do que quando o alvo era apresentada em um arranjo horizontal $($ TR médio $=471$ ms) $\left(F(1,12)=5,65 ; p<0,05 ; \eta^{2}=0,32\right)$. Este resultado pode sugerir um descarte mais eficiente da informação irrelevante apresentada no meridiano vertical, ou uma concentração maior de recursos atentivos ao longo do meridiano horizontal.

Uma segunda análise, que levou em conta apenas as condições com a dica orientada (vertical e horizontal), na qual as letras flanqueadoras eram apresentadas dentro ou fora da dica, confirmou uma facilitação na seleção da letra alvo quando as letras flanqueadoras eram apresentadas fora da dica (TR médio $=481 \mathrm{~ms}$ ) do que quando apresentadas dentro da dica (TR médio $=487 \mathrm{~ms}$ ) $\left(F(1,12)=7,12, p<0,05, \eta^{2}=0,37\right)$.

Nesta situação, houve um efeito significativo do fator compatibilidade com um TR menor para letras flanqueadoras compatíveis (TR médio $=474 \mathrm{~ms}$ ) do que para letras flanqueadoras incompatíveis (TR médio $=494 \mathrm{~ms}$ ) $\left(F(1,12)=36,22, p<0,05, \eta^{2}=0,75\right)$, o que corrobora o resultado de outras pesquisas com letras flanqueadoras (B. Eriksen \& Eriksen, 1974; Miller, 1991). Novamente, a orientação do arranjo das letras (horizontal e vertical) apresentou um efeito significativo no desempenho dos participantes que apresentaram um TR maior para a seleção da letra alvo no arranjo horizontal (TR médio= $487 \mathrm{~ms})$ do que no arranjo vertical (TR médio $=482)$ $\left(F(1,12)=5,20, p<0,05, \eta^{2}=0,30\right)$.

Houve também uma interação significativa entre o fator posição das letras flanqueadoras e compatibilidade $\left(F(1,12)=10,39, p<0,01, \eta^{2}=0,46\right)$. Esta análise mostrou que as letras flanqueadoras incompatíveis tiveram um efeito maior quando apresentadas dentro da dica $(\mathrm{TR}=$ $500 \mathrm{~ms}$ ), do que quando apresentadas fora da dica (TR= $488 \mathrm{~ms})\left(F(1,12)=15,56, p<0,01, \eta^{2}=0,56\right)$. A Figura 4 mostra os TRs médios em função do tipo de dica (controle, horizontal, vertical); orientação do arranjo de letras (horizontal, vertical) e compatibilidade entre letra alvo e letras flanqueadoras (compatível e incompatível).

\section{Discussão}

Este resultado mostra que o tempo de reação médio dos participantes é prejudicado pela presença da letra franqueadora incompatível, e que este efeito é significativamente maior quando os estímulos incompatíveis são apresentados dentro da dica visual. Este resultado pode indicar que os recursos atentivos estão distribuídos desigualmente dentro e fora da dica visual, e que além do fator distância entre a letra alvo e as letras flanqueadoras apontado por Miller (1991), a presença de um limite físico entre o alvo e as letras flanqueadoras contribui significativamente para uma redução do efeito deletério causado pelas letras flanqueadoras incompatíveis. Há duas possíveis interpretações para a ação da atenção na seleção da informação relevante nesta situação. Na primeira interpretação a atenção poderia atuar na ativação de uma área delimitada pelas bordas da dica. Assim os recursos atentivos estariam restritos a esta área permi- 

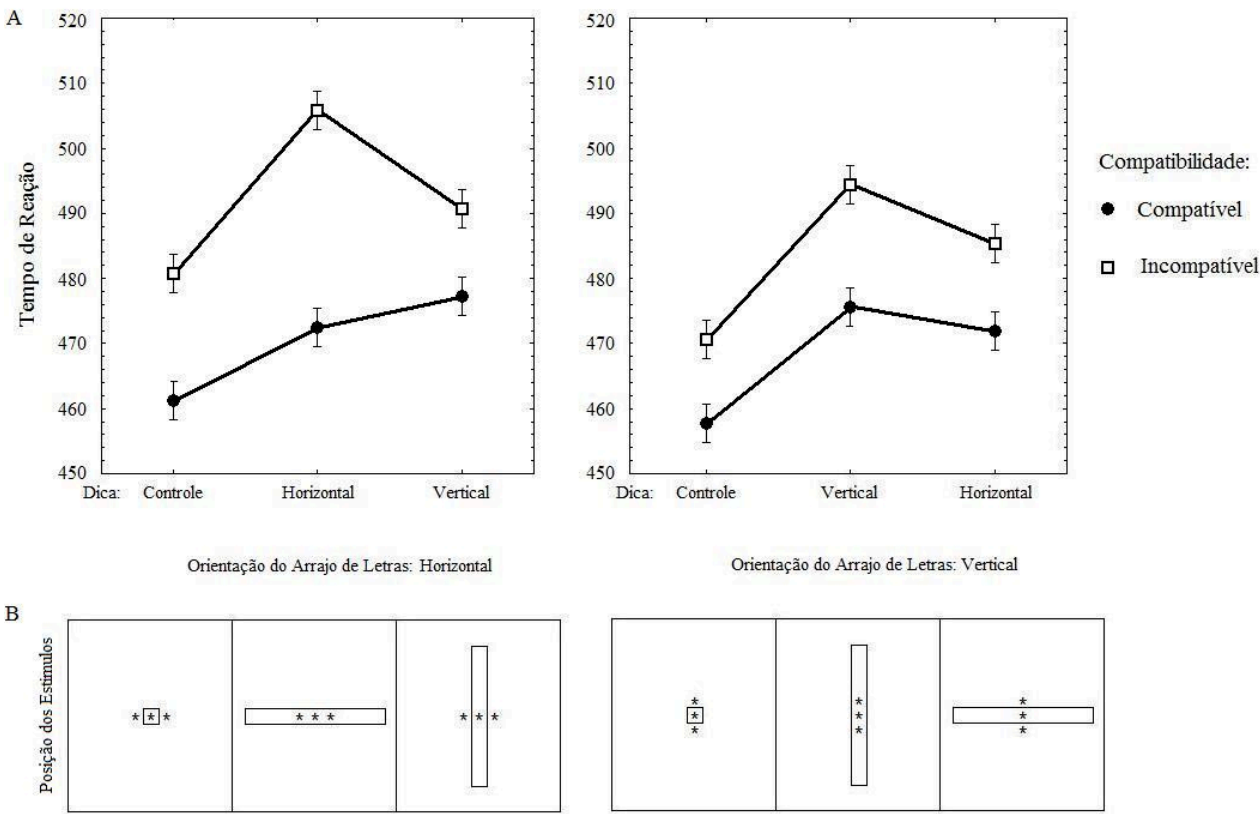

Figura 4. A- Tempo de reação médio (TR) em função do tipo de dica (controle, horizontal, vertical); orientação do arranjo de letras (horizontal, vertical) e compatibilidade entre letra alvo e letras flanqueadoras (compatível e incompatível). B- Representação esquemática dos tipos de dica e da posição ocupada pelos arranjos de letras orientadas

tindo um processamento da informação relevante mais eficiente. Uma segunda interpretação, no entanto, poderia considerar a atenção como um recurso inibitório sobre o campo irrelevante na seleção da informação. Nesta interpretação a atenção atuaria na supressão da informação apresentada fora da área da dica, o que facilitaria o processamento da informação relevante apresentada dentro da área delimitada pela dica visual (Facoetti \& Molteni, 2000).

De maneira geral, os resultados deste experimento sugerem que a atenção pode ser orientada sobre uma área delimitada do campo visual e que a presença de um limite físico, entre a letra alvo e as letras flanqueadoras incompatíveis, contribui de maneira significativa para a diminuição do seu efeito deletério na tarefa de discriminação. Tal resultado pode indicar que o foco atentivo pode, de fato, assumir um formato mais flexível do que foi originalmente proposto.

\section{Discussão Geral e Conclusão}

O presente estudo investigou dois aspectos importantes do processo de focalização dos recursos atentivos em uma área visual delimitada no espaço. No primeiro experimento investigamos o tempo necessário para a focalização e manutenção dos recursos atentivos em função do tamanho da área a ser focalizada. No experimento 2 , investigamos a distribuição dos recursos atentivos disponibilizados dentro e fora do foco, tanto no meridiano vertical quanto horizontal do campo visual.

O resultado geral do primeiro experimento é compa- tível com o modelo zoom proposto por C. Eriksen e colaboradores (C. Eriksen \& St. James, 1986; C. Eriksen \& Yeh 1985). Benso et al. (1998) obtiveram resultados que mostram que a focalização dos recursos atentivos em uma tarefa de detecção simples pode ocorrer em períodos inferiores a $100 \mathrm{~ms}$ de exposição de uma dica visual exógena, mantendo-se estável até intervalos próximos a $600 \mathrm{~ms}$. Os resultados observados no primeiro experimento mostram que o efeito do tamanho da dica em uma tarefa de discriminação simples é significativo após um intervalo de exposição de $360 \mathrm{~ms}$, muito além do tempo necessário para a focalização dos recursos em uma tarefa de detecção simples (Benso et al., 1998). Este resultado sugere que o tipo de tarefa a ser realizada pelo participante pode influenciar no tempo necessário para o ajuste dos recursos atentivos no campo visual. Os resultados do primeiro experimento também sugerem uma possível relação inversa entre o tamanho do foco e o tempo pelo qual ele pode ser mantido. Desta forma, os recursos atentivos seriam mantidos focalizados por mais tempo em pequenas áreas do que em áreas maiores de processamento.

Os resultados do segundo experimento mostraram que o efeito negativo provocado pela apresentação de letras flanqueadoras incompatíveis é significativamente menor quando estas são apresentadas fora da área delimitada pela dica visual. Esta redução do efeito deletério causado pelas letras flanqueadoras incompatíveis na seleção do alvo está vinculado à apresentação de um limite físico entre o alvo e a letra flanqueadora, o que sugere que os recursos atentivos são distribuídos na área definida 
pelas margens da dica visual. Concomitantemente a este processo, a diminuição do efeito deletério das letras flanqueadoras incompatíveis apresentadas fora da dica aponta para uma possível ação inibitória da atenção sobre a informação apresentada em uma área não relevante para a tarefa de seleção. Nesta situação a atenção teria um papel ativo na inibição da informação não relevante fora da área a ser atendida. Estes resultados corroboram o modelo proposto por C. Eriksen e colaboradores (C. Eriksen \& St. James, 1986; C. Eriksen \& Yeh 1985) com características essencialmente ativadoras, mas indicam a possibilidade de uma ação inibitória da atenção fora da área focalizada, como sugerido por Facoetti e Molteni (2000).

Finalmente, a seleção da letra alvo foi mais eficiente nas condições em que o arranjo de letras era apresentado no meridiano vertical do que no horizontal. Este resultado mostra um aspecto importante do processo de seleção da informação e da disponibilidade dos recursos atentivos no campo visual, sobretudo em tarefas que envolvam a discriminação de letras. Os resultados sugerem que é mais fácil descartar a informação irrelevante apresentada em um arranjo vertical do que em um arranjo horizontal. Talvez esta assimetria na eficiência no processo de inibição da informação irrelevante durante a seleção da informação esteja relacionada diretamente ao processo de leitura, no qual a capacidade de inibir a informação irrelevante apresentada acima e abaixo da linha focalizada desempenhe um papel fundamental para a proficiência neste tipo de tarefa.

\section{Referências}

Benso, F., Turatto, M., Mascetti, G. G., \& Umiltà, C. (1998). The time course of attentional focusing. European Journal of Cognitive Psychology, 10, 373-388.

Castiello, U., \& Umiltà, C. (1990). Size of the attentional focus and efficiency of processing. Acta Psychologica, 73, 195209.

Egly, R., \& Homa, D. (1984). Sensitisation in the visual field. Journal of Experimental Psychology: Human Perception and Performance, 10, 778-793.

Eriksen, B., \& Eriksen, C. (1974). Effects of noise letters upon the identification of a target letter in a nonsearch task. Perception \& Psychophysics, 16, 143-149.

Eriksen, C., \& St. James, J. (1986).Visual attention within and around the field of focal attention: A zoom lens model. Perception \& Psychophysics, 40, 225-240.

Eriksen, C., \& Yeh, Y. Y. (1985). Allocation of attention in the visual field. Journal of Experimental Psychology: Human Perception and Performance, 11, 583-597.

Facoetti, A., \& Molteni, M. (2000). Is attentional focusing an inhibitory process at distractor location? Cognitive Brain Research, 10, 185-188.

Galera, C., \& von Grünau, M. (2003). Size and shape of the attention spotlight affect efficiency of processing [Abstract]. Proceedings 3rd Vision Science Society Annual Meeting, 162.

Galera, C., von Grünau, M., \& Panagopoulos, A. (2005). Automatic focusing of attention on object size and shape. Psicologica (España), 26, 147-160.
Juola, J. F., Bouwhuis, E. E, Cooper, C., \& Warner, B. (1991). Control of attention around the fovea. Journal of Experimental Psychology: Human Perception \& Performance, 17, 125-141.

Maringelli, F., \& Umiltà, C. (1998). The control the attentional focus. European Journal of Cognitive Psychology, 10, 225246.

Miller, J. (1991). The flanker compatibility effect as a function of visual angle, attentional focus, visual transients, and perceptual load: A search for boundary conditions. Perception \& Psychophysics, 49, 270-288.

Panagopoulus, A., von Grünau, M. W., \& Galera, C. (2004). Attentive mechanisms in visual search. Spatial Vision, 17, 353-371.

Posner, M., Snyder, C., \& Davidson, B. (1980). Attention and detection of signals. Journal of Experimental Psychology: General, 109, 160-174.

Sanders, A. F., \& Brück, R. (1991). The effect of presentation time on the size of the visual lobe. Bulletin of the Psychonomic Society, 29, 206-208.

Schneider, Eschman, \& Zuccoloto (2002). E-prime 1.0 [Computer software]. Pittsburgh, PA: Psychological Software.

Turatto, M., Benso, F., Facoetti, A., Galfano, G., Mascetti, G. G., \& Umiltà, C. (2000). Automatic and voluntary focusing of attention. Perception \& Psychophysics, 62, 935-952.

Usai, M. C., Umiltà, C., \& Nicoletti, R. (1995). Limits in controlling the focus of attention. European Journal of Cognitive Psychology, 7, 411-439.
Recebido: 04/06/2008 $1^{a}$ revisão: $11 / 11 / 2008$ $2^{a}$ revisão: 03/02/2009 Aceite final: 06/02/2009 